\title{
Length Measurement of Pipes with Open Ends Under Noisy Environment Using Stationary Wave
}

\author{
S. Tanaka, M. Okamoto and M. Nakayama \\ Faculty of Engineering, Yamaguchi University \\ 2-16-1 Tokiwadai, Ube, Yamaguchi, JAPAN \\ tanaka@sens.eee.yamaguchi-u.ac.jp
}

\begin{abstract}
The authors previously developed a dynamic model based accurate pipe length measurement system using stationary waves generated spontaneously or by hitting the pipe. However, two problems rise when the system is applied to the length measurement of steel pipes at factories. The first is that a considerably large noise is continuously generated around the measuring spot and thus it causes low $\mathrm{SN}$ ratio in the observation. The second is that high order modes of the stationary wave cannot be available because the diameter of the steel pipes is relatively large compared to the pipe length. This paper thus proposes an accurate length measurement of a pipe under noisy environment by considering the selections of an optimal sensor location and an optimal data window.
\end{abstract}

\section{Introduction}

In forwarding steel pipes at factories, pipe lengths have to be exactly measured. For this aim, two operators have measured the pipe length with a tape measure until now. This procedure needs however much time and a large measurement error due to slacks and bending of the measure. Thus, a fast and accurate pipe length measurement system has been desired.

As an indirect measurement for the pipe length, a range sensor of light wave is known. But, in the usage of the method, a reflection board of the light has to be set at another end of the pipe and the cost of the measurement system becomes expensive. As an alternative, pulse-echo method [1], well known as the method using a sound wave, is considered to be used. But, it is not adequate to accurately measure the length under noisy environment, because the accurate measurement of time-of-flight(TOF) is very difficult.

From the viewpoints, the authors previously proposed an accurate pipe length measurement system using stationary wave generated in the pipe spontaneously or hitting the pipe [2]. The system adopted the values and the derivatives of several modes of stationary wave as the state variables of a linear dynamic system and applied a Kalman filter and a maximum likelihood method to estimate the parameter $L$ of the pipe length. Moreover, in order to realize a much more accurate length measurement of a pipe, the paper furthermore gave a selection method of the optimal modes of the stationary wave and realized an extremely accurate pipe length measurement of the error of $2 \sim 5 \mathrm{~mm}$ for the pipe of about 10 meters long.

The paper [2] showed that using the optimal modes whose amplitudes and mode numbers were both large enabled an accurate pipe length measurement for thin pipes under a silent condition. However, an extremely large noise is continuously generated in factories. Thus one approach we can consider would be to broaden in order to realize an accurate length measurement. But, the noise frequently affecting amplitudes and phases of the stationary waves in the pipe, the broadening a data window is not necessarily effective. In addition to it, steel pipes made in factories generally having large diameters compared to the pipe length, high order modes of the stationary wave cannot be used for the measurement.

To overcome the problems, the paper newly proposes an accurate pipe length measurement system for the pipe with open ends under a noisy environment. First, the optimal sensor location is derived by considering the divergence criterion. Second, the determination of the optimal data window is presented by using FFT. Finally, experimental results will show that a highly accurate length measurement of the pipes with open ends and large diameters can be realized with the proposed method.

\section{Pipe Length Measurement Based on a Linear Dynamic System Model of Stationary Wave}

\subsection{Linear dynamic system model description of the sound fluctuation in the pipe}

We consider a straight pipe of length $L$ with two open ends as shown in Fig.1. Defining the distance along the pipe axis from the left end of the pipe as $\ell$, the sound fluctuation at the point $\ell$ for the $j$-th mode of 


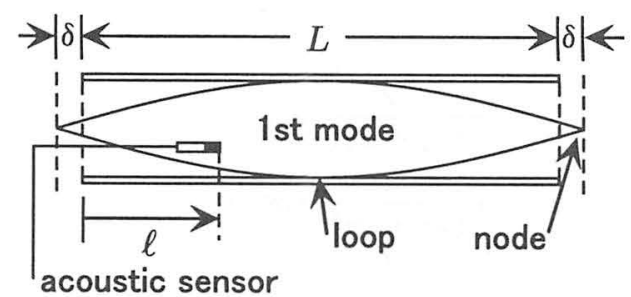

Fig.1 A straight pipe with two open ends.

the stationary wave is given by:

$$
\begin{aligned}
z_{j}(\ell, t) & =\beta_{j}(\ell) \sin \left(\omega_{j} t+\phi_{j}\right) \\
\beta_{j}(\ell) & =\alpha_{j} \sin \{\pi(\ell-\delta) /(L+2 \delta)\}
\end{aligned}
$$

where $\beta_{j}(\ell)$ and $\alpha_{j}$ are respectively the amplitudes of the $j$-th mode at the point of $\ell$ and at the point of loop of the stationary wave. Furthermore, $\omega_{j}$ represents the angular frequency of the $j$-th mode and is given as $\omega_{j}=j \times 2 \pi q /\{2(L+2 \delta)\}(j=1,2, \cdots)$, where $q$ and $\delta$ are respectively a sound velocity and an open end compensation [3]. Note that a pipe length measurement is reduced to the estimation of the angular frequency of the first mode of the stationary wave. For simplicity of discussion, the length parameter $L+2 \delta$ is defined as $L$ in the sequel. For reference, spacial distribution of the sound pressure of the 1st mode is shown in Fig.1.

Defining the set of mode numbers (to be used) as $\Omega$, the observation $y(\ell, t)$ given by an acoustic sensor set at the point $\ell$ is represented as:

$$
y(\ell, t)=\sum_{j \in \Omega} z_{j}(\ell, t)+v(\ell, t)
$$

where $v(\ell, t)$ represents the sum of unused modes of the stationary wave and an intrinsic sensor noise. We regard $v(\ell, t)$ as an observation noise. The noise including a lot of modes of the stationary wave, we can assume the noise as a white Gaussian noise with the mean zero and the variance $V(\ell, \Omega)[2]$.

Similarly in the previous measurement system[2], adopting the mode set $\Omega=\left\{i_{1}, i_{2}, i_{3}\right\}$ and defining the state vector as $x(\ell, t)=\left(z_{i_{1}}, \dot{z}_{i_{1}}, z_{i_{2}}, \dot{z}_{i_{2}}, z_{i_{3}}, \dot{z}_{i_{3}}\right)^{T}$, the following state equation is obtained.

$$
\dot{x}(\ell, t)=A x(\ell, t)+w(t)
$$

where

$$
\left.\begin{array}{l}
A=\left[\begin{array}{ccc}
A_{1} & O & O \\
O & A_{2} & O \\
O & O & A_{3}
\end{array}\right] \\
A_{j}=\left[\begin{array}{cc}
0 & 1 \\
-\omega_{i_{j}}^{2} & 0
\end{array}\right] \quad(j=1,2,3)
\end{array}\right\}
$$

where $\boldsymbol{w}(t)=\left(0, w_{1}(t), 0, w_{2}(t), 0, w_{3}(t)\right)^{T}$ is a transition noise vector which is introduced to compensate for a possible slight attenuation in a data window. We assume that the noise $w_{j}(t)(j=1,2,3)$ are independent white Gaussian noises with means zero and same variance $\sigma^{2}$.
Using the state vector $\boldsymbol{x}(\ell, t)$, the observation of $(3)$ is rewritten as:

$$
y(\ell, t)=H x(\ell, t)+v(\ell, t)
$$

where $H=[1,0,1,0,1,0]$.

Consequently, the linear dynamic system of the stationary wave is obtained as (4) and (6).

\subsection{Accurate pipe length measurement}

Discretizing (4) and (6) with a sampling period, the estimation of the state vector is executed by a discrete Kalman filter [5], since both the observation and transition noises have white Gaussian properties. However, pipe length $L$ and observation noise variance $V(\ell, \Omega)$ being unknown, Kalman filter cannot be directly applied.

Thus, defining an unknown parameter vector $\theta$ $=(L, V(\ell, \Omega))^{T}$, the Kalman filter can be applied by assigning adequate values to the vector $\boldsymbol{\theta}$. Consequently, the pipe length measurement is seen to be reduced to the optimization of the following likelihood functinon.

$$
J(\boldsymbol{\theta})=\prod_{k=1}^{M} p\left(y_{k}(\ell) / \theta, Y^{k-1}\right)
$$

where $y_{1}(\ell), y_{2}(\ell), \cdots, y_{M}(\ell)$ are time series of the observation, and $p\left(y_{k}(\ell) / \theta, Y^{k-1}\right)$ represents a conditional probability density function with respect to $y_{k}(\ell)$ under the observation $Y^{k-1} \triangleq\left\{y_{j}(\ell) ; 0 \leq j \leq\right.$ $(k-1)\}$ and the parameter vector $\boldsymbol{\theta}[2]$. Also, $\bar{M}$ is a number of the observation data to be used for the measurement. Consequently, maximizing (7) with respect to $\theta$ achieves the pipe length measurement. In order to realize an accurate length measurement of pipe and also to reduce the computational time, Powell method is used. This method offers the sequential one-dimensional search in unknown parameter space.

\section{Determination of the Optimal Sensor Location}

In this section, we discuss about an optimal sensor location from the divergence point of view.

Defining now the sensor location at $\ell$ and the mode set $\Omega$, the observation $y_{k}(\ell)$ at the sampling time $k \Delta T$ is represented as follows similar to the equation (6).

$$
\begin{array}{r}
y_{k}(\ell)=\sum_{j \in \Omega}\left\{\beta_{j}(\ell) \sin \left(\omega_{j} k \Delta T+\phi_{j}\right)\right\}+v_{k}(\ell) \\
(k=1,2, \cdots)
\end{array}
$$

where $\beta_{j}(\ell)$ and $v_{k}(\ell)$ are respectively the amplitude of the $j$-th mode of the stationary wave at the point $\ell$ and the observation noise. The noise is the sum of an intrinsic sensor noise and the unused modes of the stationary wave and thus assumed to be a white Gaussian noise similarly as in the section 2 . 
Defining now the observation vector $y(\ell)$ as

$$
\boldsymbol{y}(\ell)=\left(y_{1}(\ell), y_{2}(\ell), \cdots, y_{M}(\ell)\right)^{T}
$$

the probability density function of $y(\ell)$ is given as:

$$
\begin{aligned}
p(\boldsymbol{y}(\ell))= & \frac{1}{\{2 \pi V(\ell, \Omega)\}^{M / 2}} \\
& \times \exp \left[-\frac{1}{2} \sum_{k=1}^{M} \frac{\left\{y_{k}(\ell)-\zeta_{k}(\ell)\right\}^{2}}{V(\ell, \Omega)}\right]
\end{aligned}
$$

where

$$
\zeta_{k}(\ell)=\sum_{j \in \Omega}\left\{\beta_{j}(\ell) \sin \left(\omega_{j} k \Delta T+\phi_{j}\right)\right\}
$$

Replacing the space average as the time average, observation noise variance $V(\ell, \Omega)$ is approximated as:

$$
\begin{aligned}
V(\ell, \Omega) & =(1 / T)\left[\int_{0}^{T} \sum_{j \notin \Omega} \beta_{j}(\ell) \sin ^{2}\left(\omega_{j} t+\phi_{j}\right) d t\right]+R \\
& =(1 / 2) \sum_{j \notin \Omega}\left(\beta_{j}(\ell)\right)^{2}+R
\end{aligned}
$$

where $T=M \Delta T$ and $R$ denotes the variance of the intrinsic sensor noise.

The resolution ability with respect to the pipe length under the conditions of the sensor set at the point $\ell$ and the mode set $\Omega$ is quantified by the divergence as follows [6].

$$
D(\ell, \Omega)=\Delta L^{2} \int\left[\frac{\partial}{\partial L} \ell n p(\boldsymbol{y}(\ell))\right]^{2} p(\boldsymbol{y}(\ell)) d \boldsymbol{y}(\ell)
$$

Substituting the equation (10) into (13) gives the following equation.

$$
\begin{aligned}
D(\ell, \Omega)= & \Delta L^{2} \int \sum_{k=1}^{M}\left[\left\{\frac{\partial \zeta_{k}(\ell)}{\partial L}\left\{y_{k}(\ell)-\zeta_{k}(\ell)\right\} / V(\ell, \Omega)\right]^{2}\right. \\
& \times p(y(\ell)) d y(\ell) \\
= & \Delta L^{2} \sum_{k=1}^{M}\left[\left\{\frac{\partial \zeta_{k}}{\partial L}\right\}^{2} / V(\ell, \Omega)\right]
\end{aligned}
$$

Considering that the sampling period $\Delta T$ is sufficiently small, (14) can be approximated, by replacing the sum with respect to $k$ as the time integral over $[0, T]$, as the following.

$$
\begin{aligned}
D(\ell, \Omega) \simeq \Delta L^{2} & \sum_{i \in \Omega} \sum_{j \in \Omega}\left[i j \beta_{i}(\ell) \beta_{j}(\ell)\right. \\
& \left.\times \gamma\left(\omega_{i}, \omega_{j}\right)\left\{\frac{\partial \omega_{1}}{\partial L}\right\}^{2} / V(\ell, \Omega)\right]
\end{aligned}
$$

where $\partial \omega_{1} / \partial L=-\pi v / L^{2}$. Moreover, $\gamma\left(\omega_{1}, \omega_{2}\right)$ in (15) is given by the integral operation for the two angular frequencies $\omega_{1}$ and $\omega_{2}$ as follows.

$$
\begin{aligned}
\gamma\left(\omega_{1}, \omega_{2}\right) & =\int_{0}^{T} t^{2} \cos \left(\omega_{1} t+\phi_{1}\right) \cos \left(\omega_{2} t+\phi_{2}\right) d t \\
& =\frac{T^{2}}{\omega_{1}+\omega_{2}} \sin \left\{\left(\omega_{1}+\omega_{2}\right) T+\left(\phi_{1}+\phi_{2}\right)\right\}
\end{aligned}
$$

$$
\begin{aligned}
& +\frac{2 T}{\left(\omega_{1}+\omega_{2}\right)^{2}} \cos \left\{\left(\omega_{1}+\omega_{2}\right) T+\left(\phi_{1}+\phi_{2}\right)\right\} \\
& -\frac{2}{\left(\omega_{1}+\omega_{2}\right)^{3}} \sin \left\{\left(\omega_{1}+\omega_{2}\right) T+\left(\phi_{1}+\phi_{2}\right)\right\} \\
& +\frac{2}{\left(\omega_{1}+\omega_{2}\right)^{3}} \cos \left\{\left(\phi_{1}+\phi_{2}\right)\right\} \\
& -\left[\frac{T^{2}}{\omega_{1}-\omega_{2}} \sin \left\{\left(\omega_{1}-\omega_{2}\right) T+\left(\phi_{1}-\phi_{2}\right)\right\}\right. \\
& +\frac{2 T}{\left(\omega_{1}-\omega_{2}\right)^{2}} \cos \left\{\left(\omega_{1}-\omega_{2}\right) T+\left(\phi_{1}-\phi_{2}\right)\right\} \\
& -\frac{2}{\left(\omega_{1}-\omega_{2}\right)^{3}} \sin \left\{\left(\omega_{1}-\omega_{2}\right) T+\left(\phi_{1}-\phi_{2}\right)\right\} \\
& \left.+\frac{2}{\left(\omega_{1}-\omega_{2}\right)^{3}} \cos \left\{\left(\phi_{1}-\phi_{2}\right)\right\}\right]
\end{aligned}
$$

If the length $T$ of the data window is sufficiently larger than the period of the first mode of the stationary wave, sine waves with different angular frequencies have orthogonal relations on the time interval. Therefore, $\gamma\left(\omega_{1}, \omega_{2}\right)$ can be approximated as follows.

$$
\gamma\left(\omega_{1}, \omega_{2}\right) \simeq T^{3} / 6
$$

Using the approximation, (15) can be rewritten as:

$$
D(\ell, \Omega)=\frac{\Delta L^{2} T^{3}}{6} \sum_{j \in \Omega}\left[\left\{j \beta_{j}(\ell)\right\}^{2} / V(\ell, \Omega)\right]
$$

In the previous paper [2], only the optimal modes are selected under the condition that the sensor is located at $\ell=0$. In the paper, on the other hand, the sensor location is added as another optimization parameter. Thus, we can obtain both the optimal sensor location and the optimal modes by simultaneous optimization of (18) with respect to $\ell$ and $\Omega$.

The criterion function (18) suggests us that we can realize an accurate pipe length measurement not only by selecting the optimal modes of larger amplitudes and higher orders but also by broadening data window length $T$. However, high order modes have generally short wave lengths and thus cannot be used in the case where a diameter of the pipe is relatively large. Thus, we cannot expect any accurate measurement only by selection of the optimal modes. On the other hand, the divergence being proportional to the cubic of $T$, we can easily guess that broadening the data window length enables us to realize a much higher accurate pipe length measurement. However, since an extremely large noise enters into the pipe constantly, we cannot always simply lengthen the data window. From the reason, we next consider the determination of an optimal data window from the given data.

\section{Determination of the Optimal Data Window}

As mentioned before, an extremely large noise enters into the pipe continuously at factories. This causes abrupt variations in the amplitude and phase of the stationary wave. The variation of amplitude can be compensated somewhat by increasing the variance of 
the transition noise in dynamic equation. But the variation of phase causes a large deterioration in the measurement due to the shift of frequencies in the stationary wave. Thus, we propose here an efficient selection method of the optimal data window below.

It is well-known that all modes of the stationary wave are given by integer multiple of the frequency of that of the 1st mode of the stationary wave. But, if some phases of certain modes slightly change by the disturbance, the peak frequencies of the modes change and thus destroy the relation of the integer multiple characteristics. Moreover, in the case where phase lead and phase lag happen to cancel to each other and peak frequencies eventually do not shift, the shape of the peaks in the frequency spectrum take gentle slopes.

From these considerations, we can conclude that the selection must be defined on the properties of integer multiple relation and shape sharpness of the peaks.

First, we define the width of the data window and the starting time of the data window as $T$ and $\tau$, respectively, and apply FFT to the data window. Then the peak frequency of the $j$-th mode of the stationary wave is rewritten as $f_{j}(T, \tau)(j=1,2, \cdots)$. For the property of the integer multiple relation, we first define the following criterion function for the mode set $\Omega$.

$$
J_{1}(T, \tau)=\left[\prod_{j \in \Omega}\left\{1-\frac{f_{j}(T, \tau)}{j f_{1}(T, \tau)}\right\}\right]^{(1 / N)}
$$

where $N$ is the number of modes used.

Next, to evaluate the sharpness of the peak, we approximate the spectrum at the neighborhood of each peak as a Gaussian distribution. That is, the spectrum around the $j$-th mode frequency of the stationary wave for the data window $[\tau, T+\tau]$ is approximated as follows:

$$
\hat{P}(f)=C_{j}(T, \tau) \times \exp \left\{-\frac{1}{2} \frac{\left(f-\mu_{j}(T, \tau)\right)^{2}}{\sigma_{j}^{2}(T, \tau)}\right\}
$$

Calculating the deviation parameter $\sigma_{j}(T, \tau)$ by least square's method, the total sharpness of every peaks whose modes are adopted can be evaluated by the following criterion function.

$$
J_{2}(T, \tau)=\left[\prod_{j \in \Omega} \sigma_{j}(T, \tau)\right]^{(1 / N)}
$$

Consequently, the optimal data window is selected by simultaneously minimizing both indices (19) and (21) with respect to $T$ and $\tau$. However, for practical use, we can adopt an alternative such that a data window which does not exceed two threshold values adequately given for the two criterion functions.

\section{Experimental Results}

Fig.2 shows an outline of the experiment. The length and diameter of the pipe were $9,545 \mathrm{~mm}$ and $485 \mathrm{~mm}$.

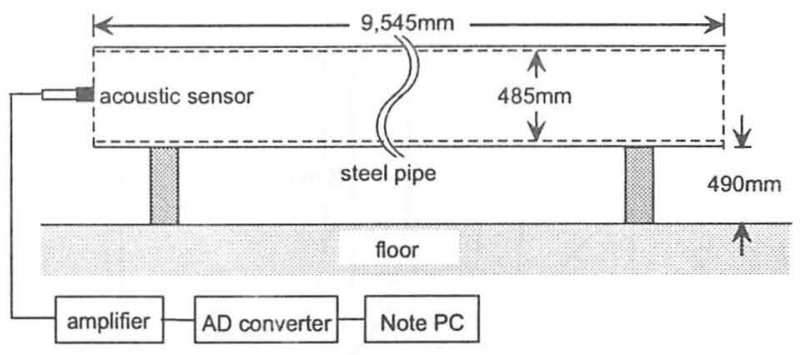

Fig.2 Outline of the experiment.

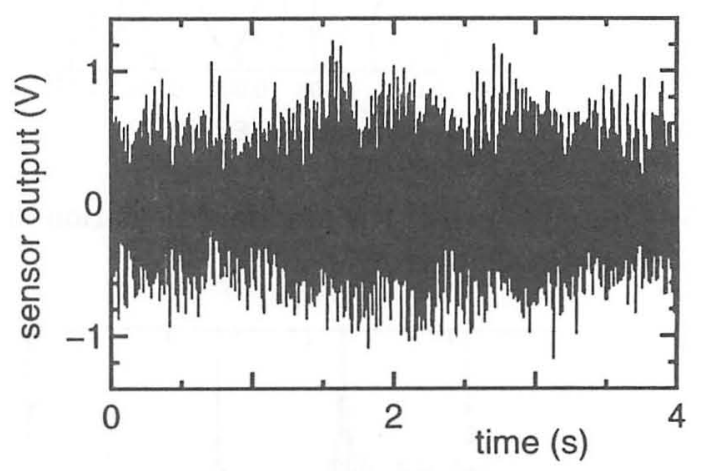

Fig.3 Sensor output.

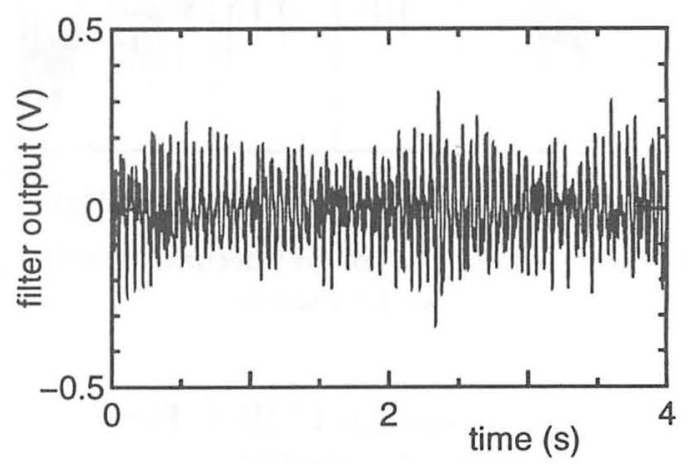

Fig.4 Output of the band-pass filter.

The temperature in the pipe when the experiment was executed was $24.4^{\circ} \mathrm{C}$. An acoustic sensor was set inside the pipe and at the end of the pipe $(\ell=0)$ and the observation data were memorized in the Note PC for $4 \mathrm{~s}$ with the sampling period $\Delta T=0.2 \mathrm{~ms}$. Note that the experiment was done only for $\ell=0$ because of the circumstances in the factory, and therefore the optimal sensor location was not considered in the experiment. The sensor output is shown in Fig.3. We can see that the output fluctuates irregularly as the time by a disturbance outside the pipe.

In the experiment, we can use only 6 modes from the 1 st to the 6 th for the measurement, since the diameter of the pipe is too large of $485 \mathrm{~mm}$. Moreover, according to the experiment, the three modes from the 1st to the 3rd were verified to be stably used for the measurement. Therefore, we determined to use only the mode set $\Omega=\{1,2,3\}$ and the other modes are all eliminated by the band pass filter with the cut off frequency of $10 \mathrm{~Hz}$ and $55 \mathrm{~Hz}$ (for reference, the frequency 


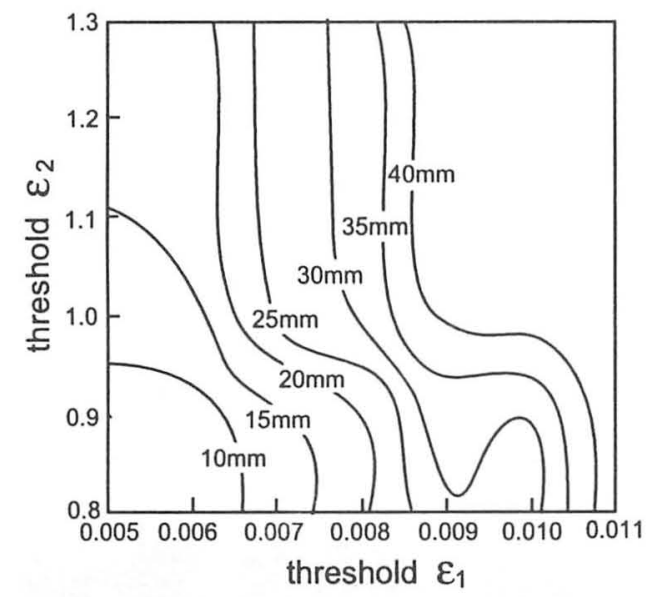

Fig.5 Contour lines of the standard deviation of the measurement error.

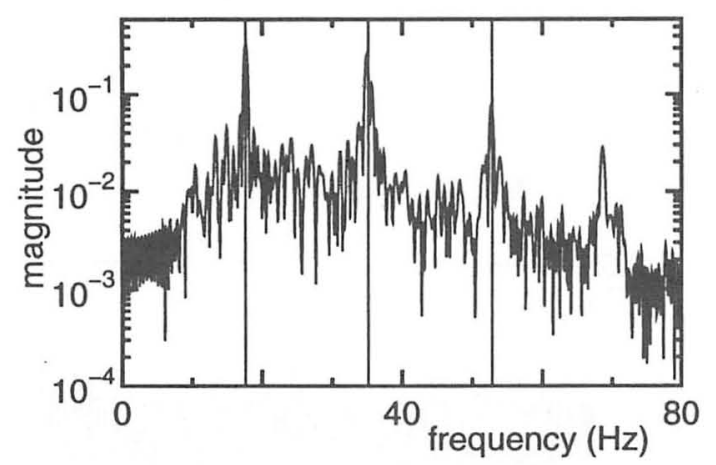

Fig.6 Spectrum of the sound fluctuation in the pipe for the optimal data window.

of the fundamental mode is $17.5 \mathrm{~Hz}$ ). Fig.4 shows the observation data after the filtering.

The proposed method is now applied to the filtered data. In order to find adequate thresholds for the criterion functions (19) and (21), we change threshold values $\varepsilon_{1}$ and $\varepsilon_{2}$ respectively in the regions of $[0.005,0.011]$ and $[0.8,1.3]$. For each pair of the thresholds, we also changed the window width three times as $1 \mathrm{~s}, 2 \mathrm{~s}$ and $3 \mathrm{~s}$. Then the proposed method is applied with the starting time of the data window optimized.

Figure 5 shows contour lines of standard deviation of the measurement error for 5 times experiments when the pair of the thresholds is changed in the region. It is seen from the figure that the standard deviation of the measurement error tends to decrease as the thresholds become small. When the pair of the thresholds is selected as $\varepsilon_{1}=0.005$ and $\varepsilon_{2}=0.8$, the standard deviation of the measurement error takes the smallest value of $5.0 \mathrm{~mm}$. In the case, mean of the pipe length measurement is $9,882 \mathrm{~mm}$ (including the open end compensation). Note that all the optimal data windows were found to have the same width $2 \mathrm{~s}$. For reference, Fig.6 shows the spectrum for an optimal data window. The vertical lines show the theoretical frequencies from the 1st to the 3rd modes of the stationary wave.

From the result, it is seen that a quite accurate pipe length measurement is realized with the proposed method even under a noisy environment and a case of large diameter of the pipe.

Finally, for reference, we try to give other data windows than the optimal data windows. For example, for the data window with width of $2 \mathrm{~s}$ and starting time of $\tau=0 \mathrm{~s}$, the standard deviation of the measurement error becomes $20.5 \mathrm{~mm}$. Moreover, for the data window with width of $4 \mathrm{~s}$ (that is, corresponds to use all the given data), the standard deviation becomes $38.0 \mathrm{~mm}$. It is seen from these facts that we cannot realize an accurate length measurement only by lengthening the width of the data window. From these results, the validity of the proposed method is verified.

\section{Conclusions}

The paper proposed an accurate pipe length measurement of the pipe with a large diameter and open ends under a noisy environment. The optimal sensor location and the determination of the optimal data window were presented. Experiments showed a highly accurate pipe length measurement with the standard deviation of $5.0 \mathrm{~mm}$ for the pipe of length $9,545 \mathrm{~mm}$ and diameter $485 \mathrm{~mm}$.

As a future work, we want to execute the experiment with the optimal sensor location.

\section{References}

[1] T.Manabe,S.Tomita and T.Yabuta : Acoustic Inspection System for Underground Telecommunication Conduit, Proc. of IMTC'94

[2] S.Tanaka and M.Okamoto : Dynamic Model Based Pipe Length Measurement Using Stationary Wave, Measurement, Vol.28, 249/259 (2000)

[3] T.Igarashi : Acoustics and Vibrations, Kyoritsu Syuppan (1968)

[4] S.Tanaka and M.Okamoto : Highly Accurate Pipe Length Measurement for Straight Pipes with Open Ends Using Stationary Waves, Journal of The Society of Instrument and Control Engineers, Vol.38,No.10,1/8 (2002)

[5] S.Arimoto: Kalman Filter, Sangyo-Tosho (1977)

[6] S.Kullback: Information Theory and Statistics, John Wiley and Sons (1959)

[7] M.Okamoto, F.Rizkyev and S.Tanaka : Optimal Location of an Acoustic Sensor for an Accurate Pipe Length Measurement Using Stationary Wave,Proc. of the 17th SICE Sensing Forum, 363/367 (2002) 